\title{
Any role left for invasive tests? Histology in clinical practice
}

\author{
P Moayyedi, M F Dixon
}

\section{Introduction}

Although some patients may consider even a venepuncture "invasive", in the context of tests for Helicobacter pylori infection this term is usually restricted to those which require gastric biopsy samples. These tests incorporate a range of techniques to show the presence of $H$ pylori in biopsy material and comprise histology, culture, rapid urease tests and, of lesser importance, microscopic examination of smears from biopsy samples or brushings and the polymerase chain reaction (PCR) which by amplifying segments of nucleic acids specific for $H$ pylori can detect very small numbers of bacteria. As only the first three are widely practised, we shall confine our consideration to them.

In comparing diagnostic tests it is conventional to examine their sensitivity - the proportion of positives that are correctly identified by the test-and their specificity - the proportion of negatives correctly identified by the testbut there are other factors influencing the general utility of a test which include availability, reproducibility, rapidity, and cost. ${ }^{1}$ Furthermore, tests vary in the amount of information they provide in addition to $H$ pylori status.

\section{Biopsy based tests} HISTOLOGY

Helicobacter pylori is one of a small select group of organisms that can be confidently recognised by histology. The histopathologist is uniquely placed both to identify infection and to assess the disease processes associated with it. Herein lies the particular advantage of histology over other $H$ pylori tests. Using a Warthin-Starry, modified Giemsa ${ }^{2}$ or Genta ${ }^{3}$ stain in addition to the routine haematoxylin and eosin, the histopathologist can recognise the typical morphology of $H$ pylori (or other helicobacter such as $H$ heilmannii ${ }^{4}$ ) and at the same time assess the pattern of gastritis and, in rare instances, recognise coincidental premalignant (dysplastic) changes or frankly neoplastic lesions such as carcinoma, lymphoma or carcinoid.

Whereas the sensitivity of histology is generally high, it will be affected by biopsy and observer related factors. There are often topographical differences in colonisation density which can introduce sampling errors. ${ }^{56} \mathrm{Al}-$ though a single biopsy from the lesser curvature $2-3 \mathrm{~cm}$ from the pylorus will yield a positive result in over $90 \%$ of infected stomachs, ${ }^{7}$ sensitivity can be improved by examining additional samples from the antrum and corpus. Corpus biopsy samples are particularly valuable when there is widespread intestinal metaplasia in the antrum as this epithelium is not colonised, or in post-treatment assessment when organisms may be cleared from the antrum but persist over corpus mucosa. ${ }^{89}$ Poor orientation or small biopsy samples will also lower sensitivity. The most important factor affecting sensitivity is the experience and conscientiousness of the observer. Cursory examination, especially when only a routine haematoxylin and eosin stain is used, will miss low density infections. ${ }^{10}$ However, even when careful examination has been undertaken in a formal observer agreement study, the concordance of results can be relatively poor. ${ }^{11}$

Specificity is generally not a problem. The characteristic morphology of $H$ pylori and its close relation to the surface epithelium render confusion with other organisms unlikely. Difficulties arise following anti-helicobacter treatment when the bacterial count may be much reduced but infection not completely eradicated. Diagnosis may rest on the proper identification of solitary bacteria. If these bacteria adopt a coccoid form then confident identification becomes impossible. It is in this situation that immunohistochemical staining, ${ }^{12}$ in situ hybridisation, ${ }^{13}$ or $\mathrm{PCR}^{14}$ may resolve the issue.

Histology is widely available in hospital practice but is relatively slow, taking at least one day to be reported. Nevertheless it is quicker than culture. Histology is an expensive test. Indeed, if the cost of endoscopy is included all invasive tests become expensive. An advantage that histology holds over other tests is the ease with which retrospective examination of slides (or new sections from archival paraffin wax blocks) can be undertaken. This can be valuable if discrepant results need to be checked. The other tests require relatively expensive storage facilities to permit retrospective analysis.

CULTURE

Culture of bacterial colonies from gastric biopsy samples which are urease, oxidase and catalase positive and on microscopic examination appear as Gram negative curved bacilli is definitive proof of $H$ pylori infection. However, the ability to isolate the organism from infected subjects varies widely between laboratories and makes it the most technically demanding of the $H$ pylori diagnostic tests. Biopsy specimens must be rapidly transferred to the laboratory in chilled transport medium, whereupon the sample is ground or minced to produce a homogenate which is inoculated onto at least two freshly prepared media, one selective and the other non-selective. Incubation occurs in a microaerobic atmosphere of $5 \%$ oxygen with $5-10 \% \mathrm{CO}_{2}$. The culture 
Table 1 Results of the various tests for Helicobacter pylor compared with the gold standard result based on the other three tests. Cases were included when one test was missing provided the other tests concurred

\begin{tabular}{llrr}
\hline & & \multicolumn{2}{l}{ Gold standard } \\
\cline { 3 - 4 } Investigation & Result & Negative & Positive \\
\hline Histology & Negative & 258 & 3 \\
& Positive & 2 & 239 \\
Rapid urease test & Negative & 262 & 22 \\
& Positive & 1 & 223 \\
Culture & Negative & 254 & 23 \\
& Positive & 2 & 218 \\
${ }^{13}$ C-UBT & Negative & 250 & 4 \\
& Positive & 3 & 231 \\
\hline
\end{tabular}

plates must be incubated at $37^{\circ} \mathrm{C}$ for at least seven days (though colonies are usually visible at four to five days). ${ }^{15}$ Only when these fastidious requirements are observed does culture compare with biopsy urease tests in its yield of positive results. These difficulties have led one prominent helicobacteriologist ${ }^{16}$ to assert that "microbiological culture these days is limited to research centres", whereas other authorities claim its general usefulness by emphasising the wide availability of culture facilities, its excellent specificity and the value of testing for susceptibility to antibiotics in patient management. ${ }^{17}$ In addition the genotype of the isolate with regard to cagA status and vacA subtype can be determined and this has a bearing on pathogenicity. ${ }^{18}$ Admittedly these latter investigations are limited to research centres.

Although totally specific if the above criteria are met, sensitivity can be affected by sampling problems, delayed transport of biopsy samples, exposure to air, and suboptimal laboratory conditions.

RAPID UREASE TESTS

The rapid urease tests use the high concentration of pre-formed urease enzyme activity in an $H$ pylori infected gastric biopsy sample to bring about a $\mathrm{pH}$ change when placed in a urea containing medium. The increasing alkalinity resulting from production of ammonium ions is detected by a colour change in the $\mathrm{pH}$ indicator. The test can be carried out using small quantities of standard urea broth for in-house methods but commercial kits, although relatively more expensive, are convenient and well standardised and are increasingly used.

The sensitivity of these rapid urease tests depends on the number of bacteria present in the biopsy. It has been calculated that $10^{4}$ organisms are required for a positive result, and a proportion of patients will harbour densities lower than this threshold. ${ }^{1}$ Low levels of colonisation are to be expected in treatment failures and the test is not sufficiently sensitive in this situation. Increased sensitivity can be achieved by using two biopsy specimens and incubation at $37^{\circ} \mathrm{C}$. Specificity is very good when the test is read at one hour but declines with the length of incubation so that at 24 hours false positive results can be given by other urease producing bacteria. ${ }^{1}$

\section{The tests compared}

We have recently completed a comparison of multiple tests for $\mathrm{H}$ pylori in dyspeptic patients attending the open access endoscopy service of the General Infirmary in Leeds. The protocol required the taking of two antral and two corpus biopsy specimens for histology, one antral biopsy sample for a rapid urease test (an in-house method using $0.5 \mathrm{ml}$ of $10 \%$ (wt/vol) unbuffered urea in distilled water to which is added one drop of $1 \%$ phenol red as the $\mathrm{pH}$ indicator) and one antral biopsy sample for culture. $\mathrm{A}{ }^{13} \mathrm{C}$-urea breath test (UBT) was performed after the endoscopy using a $4 \mathrm{~g}$ citric acid test meal and $100 \mathrm{mg}{ }^{13} \mathrm{C}$ labelled urea. Breath samples were obtained at zero and 30 minutes and were analysed using a mass spectrometer (ABCA-NT, Europa Scientific) with an excess $\delta^{13} \mathrm{CO}_{2}$ excretion of $>3.5$ being defined as positive. Patients were excluded if they had received antibiotics or bismuth salts within one month of entering the study. They were also excluded if they had received $\mathrm{H}$ pylori eradication therapy previously or they had taken proton pump inhibitors within two weeks of endoscopy. The histological $H$ pylori status was assessed (using a modified Giemsa stain) by several histopathologists working within a routine diagnostic service. All assessments were made blind to the diagnosis reached by the other tests.

The four tests were compared against each other in turn. The three other tests were used as a gold standard with two or more tests positive designated as an $H$ pylori positive patient, and all tests negative as $H$ pylori negative. If only one test was positive the subject was defined as having an indeterminate $H$ pylori status.

Five hundred and twenty patients were enrolled into the study. In 85 cases only three of the four assessments were performed but these were included in the analysis as all tests were concordant. Two patients were excluded as $H$ pylori was confined to the corpus on histology and the other invasive tests use antral biopsy samples. Nevertheless this points to the added value of histological assessment of biopsy samples from both antrum and corpus. Of the 520 patients, $245(47 \%)$ were $H$ pylori positive and $10(2 \%)$ had indeterminate status. Table 1 shows the results for histology, culture, rapid urease test, and ${ }^{13} \mathrm{C}$-UBT against the gold standard diagnoses. The one result deserving of further clarification is the presence of two false positive cultures. In the light of earlier comments on the total specificity of culture, this might be considered to be a repudiation. However, we are convinced that these two patients are indeed $H$ pylori negative and the rogue results have been attributed to a clerical error and wrong identification of specimens. We include them as this reflects actual practice in a busy endoscopy unit and laboratory.

Table 2 shows the sensitivities and specificities of the various tests. We also include the likelihood ratios which indicate the value of the test for increasing certainty about a positive diagnosis. 
Table 2 Sensitivities, specificities and likelihood ratios of the various invasive tests for Helicobacter pylori compared

\begin{tabular}{llll}
\hline Investigation & Sensitivity (\%) & Specificity (\%) & Likelihood ratio \\
\hline Histology & 98.8 & 99.2 & 124 \\
Rapid urease test & 91.0 & 99.6 & 228 \\
Culture & 90.5 & 99.2 & 113 \\
${ }^{13}$ C-UBT & 98.7 & 98.4 & 62 \\
\hline
\end{tabular}

There was little difference in the specificities of the four tests. Histology was significantly more sensitive than culture or rapid urease test (8 percentage point increase in sensitivity; $95 \%$ confidence interval 4 to 12 percentage point increase). However it should be emphasised that the latter tests were each based on a single antral biopsy samples as opposed to the four specimens examined histologically. In particular the sensitivity of culture can be improved by using multiple biopsy specimens from antrum and corpus.

\section{Histology in clinical practice}

The role of histology in the investigation and management of dyspepsia is largely a secondary issue which is subservient to the role of endoscopy. In recent years strategies have been proposed which would substantially reduce the need for endoscopy, ${ }^{19}{ }^{20}$ but gastroenterologists are reluctant to dispense with a specialised procedure which gives them the opportunity to visualise the gastroduodenal mucosa and also provides a source of income. It seems more likely that the increasing availability of rapid non-invasive ("office") tests for $H$ pylori infection, specifically whole-blood or serum based tests or even the ${ }^{13} \mathrm{C}$ UBT, will mean that primary care physicians will first try the effect of eradication treatment on symptoms before referring patients for endoscopy. If this approach is widely adopted, the frequency of endoscopy will decline. While in young dyspeptics this may be appropriate, in patients over 45 years of age endoscopy is still the investigation of choice to exclude upper gastrointestinal malignancy.

For patients undergoing upper gastrointestinal endoscopy the gastroenterologist has to decide whether the cost-benefits of histology make it worthwhile. We have already indicated that a biopsy urease test is rapid and reliable, and the $H$ pylori status can be determined in most cases before the patient leaves the endoscopy unit. This allows immediate discussion of the result with the patient and eradication therapy to be started if this is considered appropriate. Under these circumstances histology might be considered superfluous. The physician will be persuaded of the merits of histology by two factors, firstly the added value provided by the histopathologists' findings, and secondly by a fear that without histology unsuspected malignancy (or important premalignant changes) will be missed.

ADDED VALUE OF HISTOLOGY

Endoscopy itself will frequently provide a clinical diagnosis but histology is mandatory if a gastric ulcer or other potentially malignant lesion is seen. In the case of a "negative" gastroscopy the absence of a macroscopic lesion can give much-wanted reassurance to the patient. However endoscopic appearances are rarely specific and are poorly correlated with histological changes. In many cases histology will provide additional information of value in management. The nature of any gastritis (or gastropathy) present can be determined-an acute, chronic or special form, and the pattern of involvement will be an indicator of the likely disease associations. Focal gastritis will raise the possibility of Crohn's disease ${ }^{21}$; a granulomatous gastritis can also suggest Crohn's disease but might be part of some other systemic disease ${ }^{22}$; lymphocytic gastritis is associated with gluten enteropathy and lesser degrees of disturbance of small bowel function $^{2324}$ and a similar histological picture can be found in Menetrier's disease ${ }^{25}$; eosinophilic gastritis is an allergic condition which can involve other parts of the alimentary tract and has important management consequences. ${ }^{26}$ Although these are uncommon findings they would remain undiagnosed without histology. Much more frequently biopsy samples will reveal a chemical or reactive gastritis (or gastropathy) which can be a consequence of bile reflux ${ }^{27}$ or non-steroidal antiinflammatory drug use (NSAID). ${ }^{28}{ }^{29}$ Given that these drugs can be purchased over the counter, there might not always be a clear history of NSAID use and the clinician can be alerted to their potential gastrointestinal side effects. We could be more encyclopaedic in covering clinically relevant diagnoses. Suffice it to say that such histological diagnoses provide considerable added value to the simple determination of $H$ pylori status.

With regard to the topography of gastritis as determined by assessing antral and corpus biopsy specimens, in chronic $H$ pylori gastritis there is a well established relation between an antral predominant pattern and the duodenal ulcer diathesis, ${ }^{30}$ whereas a corpus predominant pattern, especially if combined with multifocal atrophy, is associated with an increased risk of gastric ulcer and carcinoma. ${ }^{31}{ }^{32}$ Furthermore, the demonstration of an $H$ pylori negative corpus restricted gastritis with diffuse corpus atrophy may alert the clinician to unsuspected pernicious anaemia. It is also worth re-emphasising that histology is the only "H pylori test" which will distinguish infection by $H$ heilmannii.

The other invasive tests are routinely carried out on antral biopsy samples alone whereas an adequate histological examination demands two antral and two corpus samples. ${ }^{33}$ In the presence of widespread antral intestinal metaplasia there may be no colonisation in antral biopsy specimens but infection is confirmed in the corpus samples. Likewise following treatment with proton pump inhibitors, infection may be cleared from the antrum but persist over corpus mucosa. In these instances the other invasive tests will give a false negative result. With the assumption that histology would be a routine investigation, the Maastricht consensus meeting advocated that "gastritis with severe abnormalities"- that is, pa- 
tients with intestinal metaplasia, atrophy, and the erosive or hypertrophic forms of gastritis, should be strongly recommended to undergo $H$ pylori eradication treatment. ${ }^{34}$ However, others would argue that such findings are largely irrelevant to management in that all patients shown to be $H$ pylori positive should be offered eradication treatment.

WILL UNSUSPECTED MALIGNANCY BE MISSED?

The one argument which could deflect gastroenterologists from abandoning routine histology would be the possibility that occult malignancies or important premalignant changes will be missed. All experienced gastroenterologists will recall one or more patients in whom a "normal" endoscopy was followed by a diagnosis of intramucosal carcinoma or high grade dysplasia in the "routine" biopsy samples. What is the likelihood of this outcome in a low cancer risk population? In order to tackle this question, an audit of biopsy diagnoses of malignancy (gastric cancer, lymphoma, carcinoid) and dysplasia over a two year period in the Leeds department was undertaken. ${ }^{35}$ There were 115 cases of malignancy and 20 cases of dysplasia among 8907 endoscopies from which gastric biopsy specimens were submitted for histology. In 128 of these cases the samples were targeted on ulcers or abnormal areas of mucosa and in six cases there was diffuse mucosal thickening. In only one case was adenocarcinoma diagnosed in a patient with a normal endoscopic appearance and this patient was undergoing "mapping" biopsies following a previous diagnosis of dysplasia at another hospital. Thus in this audit, the performance of "routine" biopsies not targeted at endoscopically visible lesions did not increase the detection of gastric malignancy or dysplasia. However, it must be emphasised that in a high risk population the yield of premalignant changes would be higher. In this situation not only are "routine" biopsy samples from the antrum and corpus advocated but they should be supplemented with a further specimen from the incisura, the site most likely to yield a diagnosis of dysplasia. ${ }^{33}$

\section{Conclusion}

In many health care systems, costs are forcing gastroenterologists and other clinicians to review critically their use of endoscopy and the ancillary tests of $H$ pylori status. Histology is a highly sensitive and specific test for $H$ pylori but is slow and expensive. However, histology also provides definitive diagnoses which carry significant consequences for patient management. Although "routine" biopsy samples are unlikely to reveal malignancy or dysplasia in low risk populations, occasional cases do occur. In high risk populations the need for histological examination is self evident. In relatively affluent health care systems managers may be persuaded that the "added value" of histology makes this a justifiable investigation. In poorer countries a rapid urease test may be more appropriate. In most Western societies we advocate, as others before us, that "gastroscopy is incomplete without biopsy". ${ }^{36}$ Whether or not this practice will prevail after rigorous cost effectiveness analyses ${ }^{37}$ has yet to be determined.

We thank Dr David Tompkins for culturing $H$ pylori, $\mathrm{Mr}$ Michael Clough and Ms Elaine Hemingbrough for performing
the ${ }^{13} \mathrm{C}-\mathrm{UBT}$. We are grateful to Cortecs Diagnostics Ltd for funding a proportion of the culture and ${ }^{13} \mathrm{C}$-UBT.

1 Mégraud F. Advantages and disadvantages of current diagnostic tests for the detection of Helicobacter pylori. Scand 7 Gastroenterol 1996;31(suppl 215):57-62.

2 Gray SF, Wyatt JI, Rathbone BJ. Simplified techniques for dentifying Campylobacter pyloridis. F Clin Pathol 1986;39: 1729-80

3 Genta RM, Robason GO, Graham DY. Simultaneous visualization of Helicobacter pylori and gastric morphology: a new stain. Hum Pathol 1994;25:221-6.

4 Heilmann KL, Borchard F. Gastritis due to spiral bacteria other than Helicobacter pylori: clinical, histological and ultrastructural findings. Gut 1991;32:137-40

5 Nedenskov-Sorensen P, Aase S, Bjorneklett A, et al. Sampling efficiency in the diagnosis of Helicobacter pylori infection and chronic active gastritis. F Clin Microbiol 1991; 29:672-5.

6 Morris A, Ali MR, Brown P, et al. Campylobacter pylori infection in biopsy specimens of gastric antrum: laboratory diagnosis and estimation of sampling error. F Clin Pathol 1989;42:727-32.

7 Genta RM, Graham DY. Comparison of biopsy sites for the histopathologic diagnosis of Helicobacter pylori: a topographic study of $\mathrm{H}$. pylori density and distribution. Gastrointest Endosc 1994;40:342-5.

8 Stolte M, Bethke B. Elimination of Helicobacter pylori under treatment with omeprazole. Z Gastroenterol 1990;28:271-4.

9 Cariani G, Vandelli A, Santini D. Antral and body Helicobacter pylori status after short- and long-term omeprazole monotherapy. Eur $\mathcal{F}$ Gastroenterol Hepatol 1992;4: $867-8$.

10 Molyneux AJ, Harris MD. Helicobacter pylori in gastric biopsies - should you trust the pathology report? $\mathcal{F} R$ Coll Physicians Lond 1993;27:119-20

11 Hojer Christensen A, Gjorup T, Hilden J, et al. Observer homogeneity in the histologic diagnosis of Helicobacter pylori. Latent class analysis, kappa coefficient, and repeat pylori. Latent class analysis, kappa coefficient, and

12 Cartun RW, Pedersen CA, Krzymowski GA, et al. Immunocytochemical detection of Helicobacter pylori in formalin fixed tissue biopsy specimens [letter]. F Clin Pathol 1990;43:518.

13 Bashir MS, Lewis FA, Quirke P, et al. In situ hybridisation for the identification of Helicobacter pylori in paraffin wax embedded tissue. f Clin Pathol 1994;47:862-6.

14 Ho S-A, Hoyle JA, Lewis FA, et al. Direct polymerase chain reaction test for detection of Helicobacter pylori in humans and animals. F Clin Microbiol 1991;29:2543-9.

15 Tompkins D. Diagnosis: Microbiological tests. In: Northfield TC, Mendall M, Goggin PM, eds. Helicobacter pylori infection: pathophysiology, epidemiology and management. London: Kluwer Academic Publishers, 1993:116-26.

16 Goodwin CS, Mendall MM, Northfield TC. Helicobacter pylori infection. Lancet 1997;349:265-9.

17 Holton J. Clinical relevance of culture: why, how and when. Helicobacter 1997;2(suppl 1):25-33.

18 Atherton J. The clinical relevance of strain types of Helicobacter pylori. Gut 1997;40:701-3.

19 Sobala GM, Crabtree JE, Pentith JA, et al. Screening dyspepsia by serology to Helicobacter pylori. Lancet 1991; 338:94-6.

20 Silverstein MD, Petterson T, Talley NJ. Initial endoscopy or empirical therapy with or without testing for Helicobacter pylori for dyspepsia: a decision analysis. Gastroenterology 1996;110:72-83.

21 Oberhuber G, Püspök A, Oesterreicher C, et al. Focally enhanced gastritis: A frequent type of gastritis in patients
with Crohn's disease. Gastroenterology 1997;112:698-706.

22 Ectors NL, Dixon MF, Geboes KJ, et al. Granulomatous gastritis: a morphological and diagnostic approach. Hisgastritis: a morphological
topathology 1993;23:55-61.

23 De Giacomo C, Gianatti A, Negrini R, et al. Lymphocytic gastritis: a positive relationship with celiac disease. $\mathcal{F}$ Pediatr 994;124:57-62

24 Wolber R, Owen D, Del Buono L, et al. Lymphocytic gastritis in patients with celiac sprue or spruelike intestinal disease. Gastroenterology 1990;98:310-15.

25 Wolfsen HC, Carpenter HA, Talley NJ. Menetrier's disease: a form of hypertrophic gastropathy or gastritis? Gastroenterology 1993;104:1310-19.

26 Caldwell JH, Sharma HM, Hurtubise PE, et al. Eosinophilic gastroenteritis in extreme allergy. Gastroenterology 1979;77: $560-4$.

27 Sobala GM, OConnor HJ, Dewar EP, et al. Bile reflux and intestinal metaplasia in gastric mucosa. F Clin Pathol 1993; 46:235-40.

28 Sobala GM, King RF, Axon AT, et al. Reflux gastritis in the intact stomach. $\mathcal{F}$ Clin Pathol 1990;43:303-6.

29 Quinn CM, Bjarnason I, Price AB. Gastritis in patients on non-steroidal anti-inflammatory drugs. Histopathology $1993 ; 23: 341-8$ 
30 Dixon MF. Helicobacter pylori and peptic ulceration: Histopathological aspects. $\mathcal{F}$ Gastroenterol Hepatol 1991;6 Histopa

31 Sipponen P. Gastric cancer - a long-term consequence of Helicobacter pylori infection? Scand f Gastroenterol 1994; 29(suppl 201):24-7.

32 Miehlke S, Bayerdörffer E, Meining A, et al. Identifying persons at risk of gastric cancer. Helicobacter 1997;2(supp 1):61-6

33 Dixon MF, Genta RM, Yardley JH, et al. Classification and grading of gastritis: The updated Sydney System. Am F Surg Pathol 1996;20:1161-81.
34 European Helicobacter pylori Study Group. Current concepts in the management of Helicobacter pylori infection. The Maastricht consensus report. Gut 1997;41:8-13.

35 Cadman B, Dixon MF, Wyatt JI. Value of routine, non-targeted biopsies in the diagnosis of gastric neoplasia. f Clin Pathol 1997;50:832-4.

36 Carpenter HA, Talley NJ. Gastroscopy is incomplete without biopsy: clinical relevance of distinguishing gaswithout biopsy: clinical relevance of distinguishing gas-
tropathy from gastritis. Gastroenterology 1995;108:917-24.

37 Fechner, RE. Value-based surgical pathology: a valuable concept. Am f Clin Pathol 1997;108:123-6. 